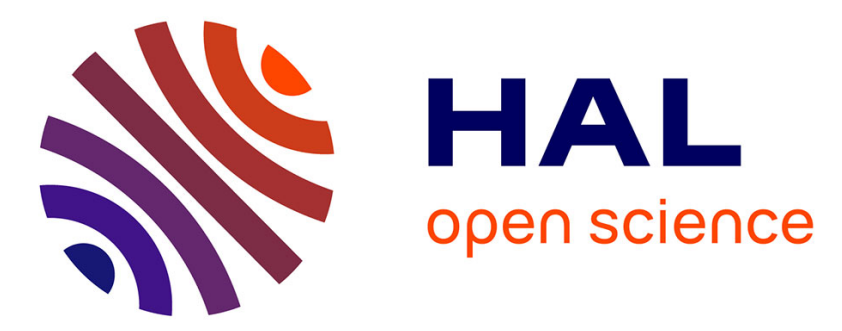

\title{
Alternate multiwavelength modelocked fiber laser
}

J. Vasseur, M. Hanna, J.M. Dudley, J.-P. Goedgebuer

\section{To cite this version:}

J. Vasseur, M. Hanna, J.M. Dudley, J.-P. Goedgebuer. Alternate multiwavelength modelocked fiber laser. IEEE Photonics Technology Letters, 2004, 16 (8), pp.1816-1818. 10.1109/LPT.2004.829774 . hal-00097101

\section{HAL Id: hal-00097101 \\ https://hal.science/hal-00097101}

Submitted on 11 May 2021

HAL is a multi-disciplinary open access archive for the deposit and dissemination of scientific research documents, whether they are published or not. The documents may come from teaching and research institutions in France or abroad, or from public or private research centers.
L'archive ouverte pluridisciplinaire HAL, est destinée au dépôt et à la diffusion de documents scientifiques de niveau recherche, publiés ou non, émanant des établissements d'enseignement et de recherche français ou étrangers, des laboratoires publics ou privés. 


\title{
Alternate Multiwavelength Modelocked Fiber Laser
}

\author{
Jérôme Vasseur, Marc Hanna, John Dudley, and Jean-Pierre Goedgebuer, Member, IEEE
}

\begin{abstract}
We demonstrate alternate mode-locking of three 30-ps pulse trains at three different wavelengths, each at a repetition rate of $3.8 \mathrm{GHz}$. The successive multiwavelength pulsed operation is reached in an actively mode-locked erbium-doped fiber laser in which an unbalanced Mach-Zehnder interferometer has been introduced. This component plays both the role of intensity modulator and tunable filter.
\end{abstract}

Index Terms-Laser tuning, mode locked lasers, optical communication, optical fiber lasers, pulsed lasers.

\section{INTRODUCTION}

$\mathbf{T}$ HE GENERATION of multicolor optical pulse trains has been intensively studied recently to obtain pulses at several wavelengths simultaneously or alternatively in the 1550-nm window. The main motivation for such sources is that the costs of current systems may be considerably reduced by using a single laser emitting simultaneously at different wavelengths. Such multiwavelength lasers are cost-effective sources for wavelength-division-multiplexing systems but also time-resolved spectroscopy and optical component characterization. In addition to being all-fiber systems, erbium-doped fiber amplifiers (EDFA) are adequate gain media for multiwavelength operation due to their wide gain curve. Several methods have been proposed to obtain multiwavelength operation in fiber lasers: the use of birefringent cavities [1] or of second-order nonlinearity with comb filters [2], the dispersion tuning method [3], the introduction of fiber Bragg gratings [4], [5] in the cavity, or more recently, the use of a frequency shifter combined with a Fabry-Pérot periodic filter [6], [7]. In this letter, we report the design and implementation of an alternate multiwavelength picosecond pulsed fiber laser that generates three pulse trains at three different wavelengths alternatively. This laser is actively mode-locked at a repetition rate of $3.8 \mathrm{GHz}$ using an unbalanced Mach-Zehnder interferometer (UMZI). The UMZI plays both the role of optical filter and intensity modulator (IM). Results obtained in a laser with both the UMZI and a conventional IM are also presented. These sources might allow the implementation of two-dimensional (2-D) wavelength-time optical code-division multiple-access (OCDMA).

\section{PRINCIPLE}

The experimental configuration of the laser is shown in Fig. 1. It consists of a gain-flattened EDFA (Keopsys model 1060 234),

J. Vasseur and M. Hanna are with GTL-CNRS Telecom, UMR CNRS 6174, 57070 Metz, France (e-mail: jvasseur@georgiatech-metz.fr; mhanna@georgiatech-metz.fr).

J. Dudley and J.-P. Goedgebuer are with Laboratoire FEMTO-ST, UMR CNRS 6174, 25030 Besançcon Cedex, France (e-mail: john.dudley@univfcomte.fr; jean-pierre.goedgebuer@univ-fcomte.fr).

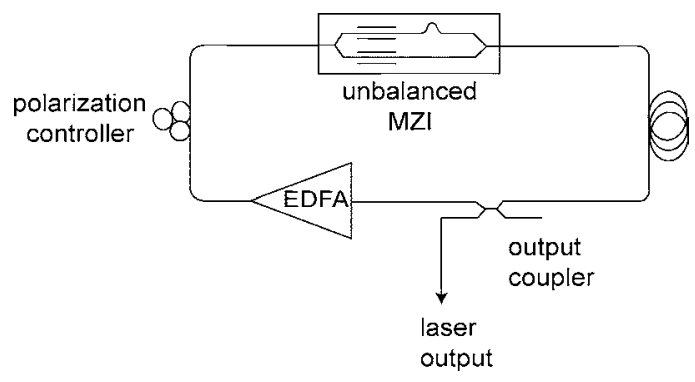

Fig. 1. Schematic diagram of the alternate multiwavelength laser.

a polarization controller, an unbalanced MZI (Photline Technologies custom order), and a 50/50 output coupler.

The cavity dispersion was measured to be less than $1 \mathrm{ps} / \mathrm{nm} / \mathrm{km}$, allowing mode-locking on a wide range of wavelengths. The UMZI is an integrated annealed proton exchanged $\mathrm{LiNbO}_{3}$ 10-Gb/s modulator that includes a fixed optical path difference of $40 \mu \mathrm{m}$ between its two arms. When no radio-frequency signal is applied onto the UMZI, it behaves as a spectral filter, with a sinusoidal frequency transfer function and a free spectral range (FSR) of $60 \mathrm{~nm}$. Upon application of a time-varying electrical signal, this transfer function shifts accordingly. Equivalently, each wavelength sees a different temporal transfer function that depends on the bias of the MZI and the frequency and modulation depth used. This results in a wavelength-dependent mode-locking condition and in an alternate generation of pulses at different wavelengths. Considering the input field amplitude $u(t)=e^{j \omega t}$, with $\omega$ in the neighboorhood of a reference angular frequency $\omega_{0}$, the transfer function of the UMZI $T$ is proportional to

$$
T \sim \cos \left(\frac{\omega \tau}{2}\right)
$$

where $\tau$ is the delay introduced by the MZI, assumed to be of the form $\tau(t)=\tau_{0}+\Delta \tau \sin (\Omega t)$. We choose the reference angular frequency, $\omega_{0}$, to be a maximum of transmission for the filter at $t=0: \omega_{0}=4 k \pi / \tau_{0}$, with $k$ an integer. Since $\tau \approx \tau_{0}$, the transfer function is maximum for the angular frequency $\omega_{\max }$ that satisfies $\omega_{\max } \tau / 2=2 k \pi$. Using a Taylor expansion, $\omega_{\max }$ can be evaluated as

$$
\omega_{\max } \approx \omega_{0}\left(1-\frac{\Delta \tau}{\tau_{0}} \sin (\Omega t)\right) .
$$

Fig. 2 shows the theoretical evolution of the maximum of the transfer function of the UMZI as a function of time (with $\tau_{0}=$ $1.3310^{-13} \mathrm{~s}, \omega_{0}=1.21610^{15} \mathrm{rad} / \mathrm{s}, \Omega=2.3810^{10} \mathrm{rad} / \mathrm{s}$, and $\Delta \tau=1.910^{-15}$ s to fit the experimental parameters). Note that a similar principle was first used by Olsson and Tang [8] in a bulk laser with separated tunable filter and mode-locker elements. 


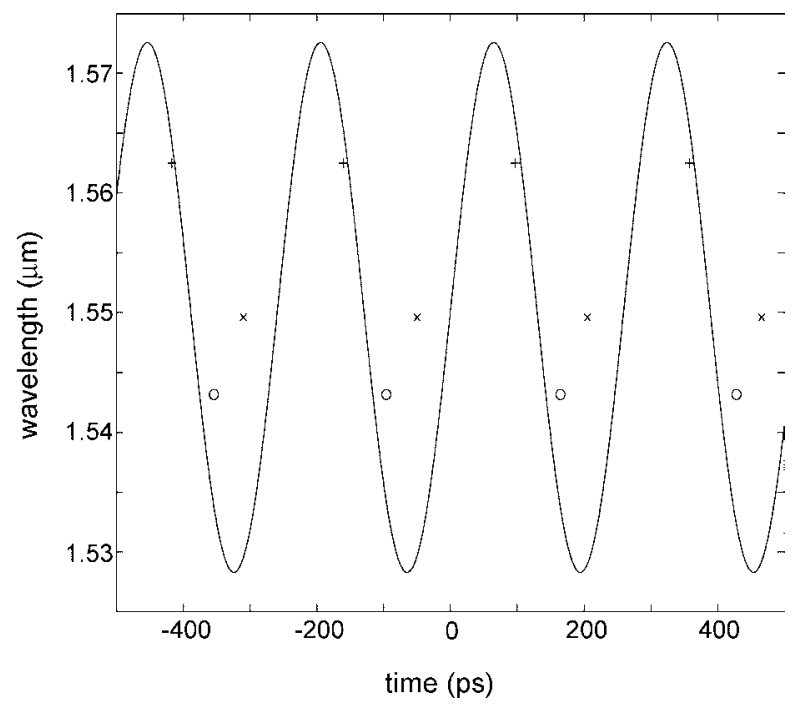

Fig. 2. Theoretical evolution of the maximum of the transfer function of the UMZI as a function of time (solid line) and experimental behaviors (points).

(a)

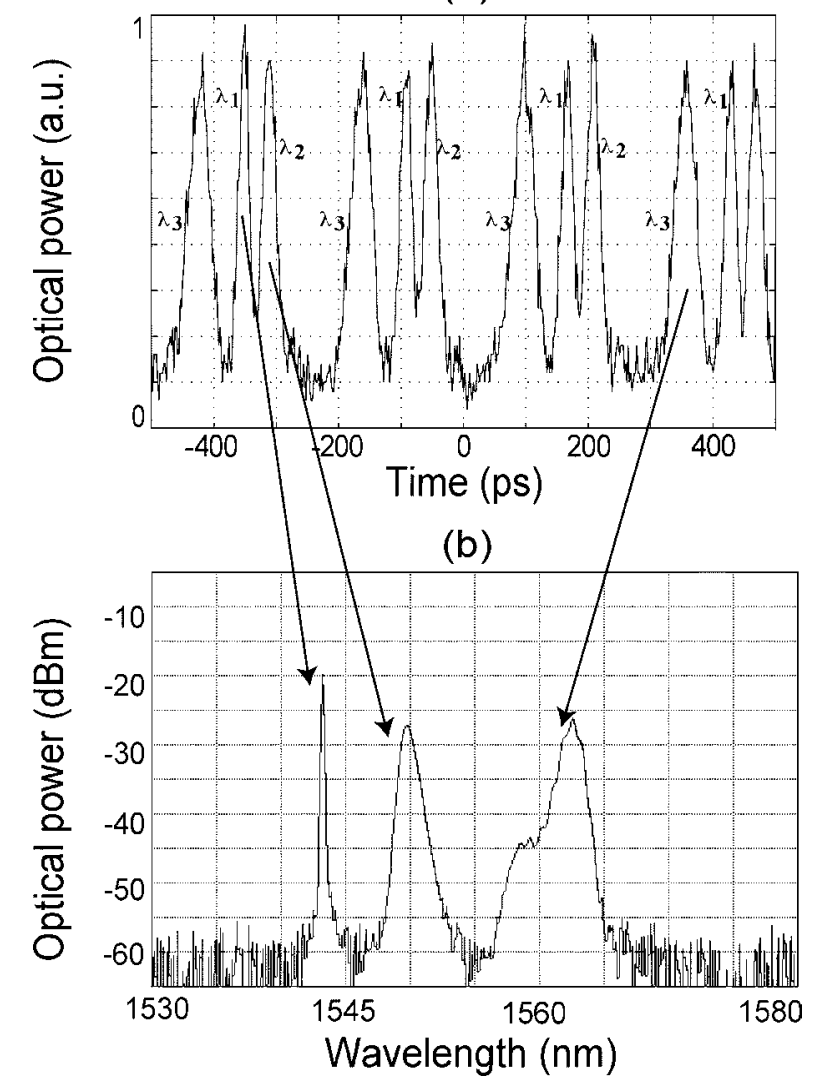

Fig. 3. (a) Time trace and (b) spectrum at the laser output.

\section{EXPERIMENTAL RESULTS}

Fig. 3(a) and (b) display the temporal trace and spectrum of the signal at laser output, respectively. For a repetition rate of $3.8 \mathrm{GHz}$, three successive trains at three different wavelengths $\left(\lambda_{1}=1543.2 \mathrm{~nm}, \lambda_{2}=1549.6 \mathrm{~nm}\right.$, and $\left.\lambda_{3}=1562.5 \mathrm{~nm}\right)$ are observed using an optical spectrum analyzer with $0.07-\mathrm{nm}$ resolution and a sampling oscilloscope with a $30-\mathrm{GHz}$ photodiode. The wavelength of each pulse is identified by adding a

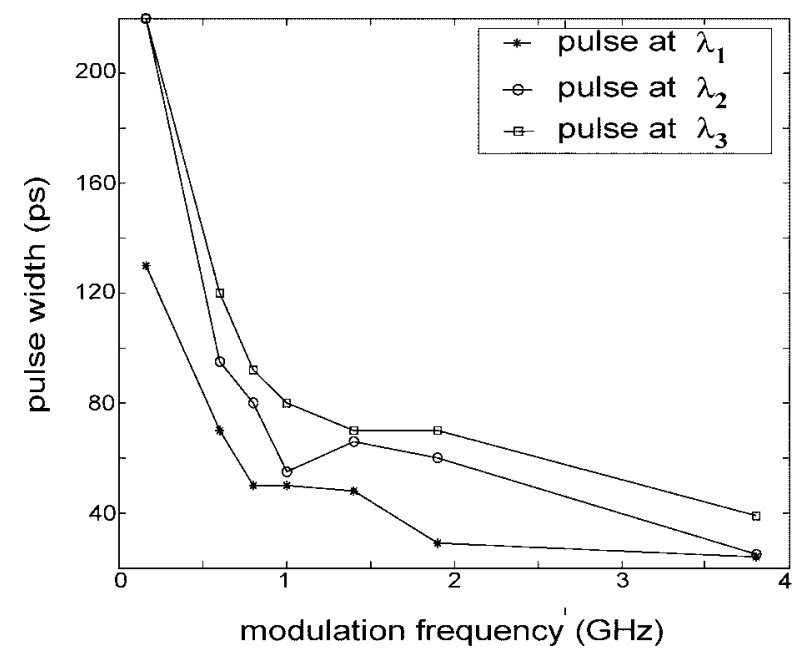

Fig. 4. Full-width at half-maximum pulsewidth as a function of mode-locking frequency for the three emitted wavelengths $\left(\lambda_{1}=1543.2 \mathrm{~nm}, \lambda_{2}=\right.$ $1549.6 \mathrm{~nm}$, and $\left.\lambda_{3}=1562.5 \mathrm{~nm}\right)$.

1.2-nm bandpass tunable optical filter at the laser output and the pulsewidths were recorded to be 24,25 , and $38 \mathrm{ps}$, yielding a time-frequency product of $0.81,1.06$, and 1.85 , respectively. Since the center wavelength of the filter varies with time, the generation of chirped pulses is expected. The three wavelengths' operation is stable for some hours at a constant room temperature. Nevertheless, since no stabilization system has been implemented, fluctuations of the peak power are observed. The time locations of these emitted wavelengths is plotted on Fig. 2. Experimental behaviors of $\lambda_{1}$ and $\lambda_{3}$ are in good agreement with the expected theoretical behavior. $\lambda_{2}$, however, is not accounted for by the filter transfer function. This might indicate that, for this filter position, the overall frequency selection is dominated by the EDFA transfer function. As shown in Fig. 4, the three pulse trains-three colors operation of the laser has been observed at different harmonics of the frequency axial mode separation for a range from some megahertz up to $3.8 \mathrm{GHz}$. As expected in a mode-locked configuration, the pulsewidth decreases with an increase of the modulation frequency [9].

Typically, the large homogeneous broadening occuring in the EDFA causes mode competition in a $10-\mathrm{nm}$ window around each laser oscillation and prevents emission of closely separated wavelengths [10], [11]. Nevertheless, as mentioned in [5], temporal-spectral multiplexing helps overcome homogeneous broadening in multiwavelength pulsed lasers. In this laser, the wide separation of the emitted wavelengths (about 6 and $12 \mathrm{~nm}$ ) seems to be inherent to the specific mode-locking technique used. The large FSR of the UMZI and the necessary adequation between the location of the pulse in the time domain and its spectral content, in the absence of any other constraint, are imposing such widely separated wavelengths. The exact emitted wavelengths are determined by local extrema of the gain curve of the EDFA.

\section{DISCUSSION}

In order to have a better control on the timing of the emitted pulses, a conventional IM, integrated on $\mathrm{LiNbO}_{3}$, is added in the 
(a)

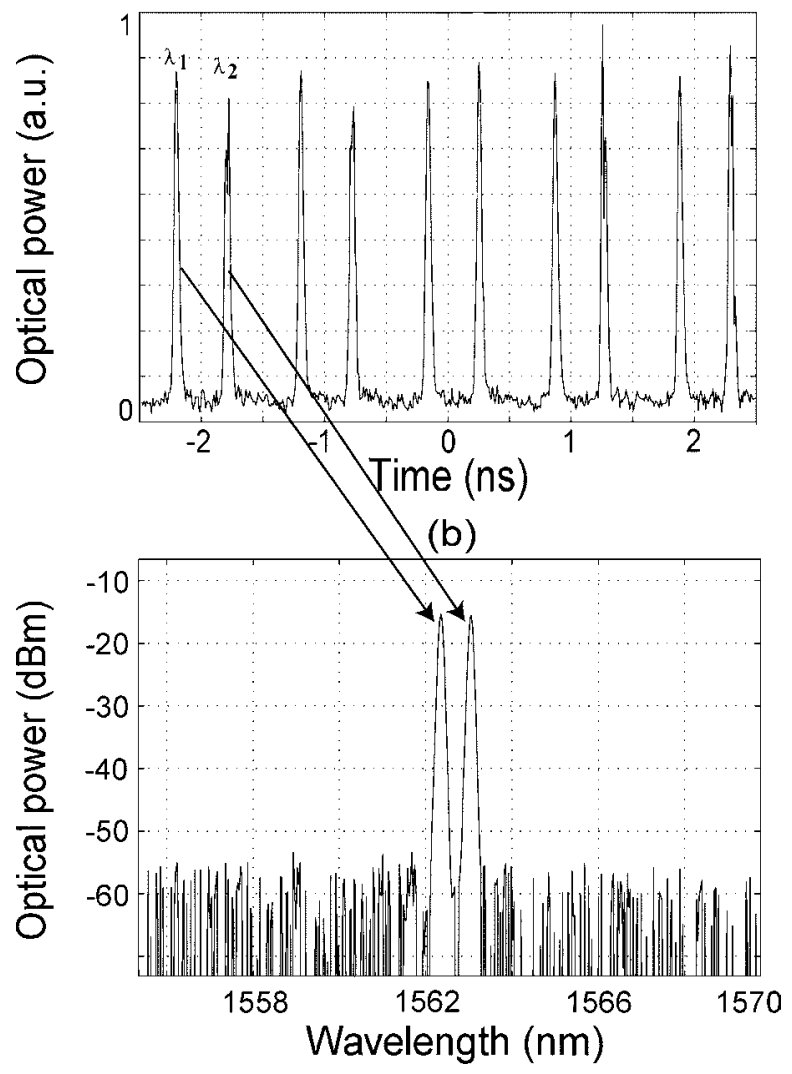

Fig. 5. (a) Time trace and (b) spectrum at the laser output in the presence of a conventional IM.

ring cavity. The IM is synchronously driven at an integer multiple of the UMZI modulation frequency. It locks the emission of pulses on a temporal grid, while the central wavelength is determined by the UMZI. Fig . 5 shows the experimental results when the modulation frequency of the $\mathrm{IM}\left(f_{\mathrm{IM}}=4.837 \mathrm{GHz}\right)$ is five times the modulation frequency of the UMZI $\left(f_{\mathrm{UMZI}}=\right.$ 967.4 MHZ).

Two series of pulses at two different wavelengths $\left(\lambda_{1}=1562.3 \mathrm{~nm}\right.$ and $\left.\lambda_{2}=1563 \mathrm{~nm}\right)$ are obtained. This confirms the possible laser emission at closely spaced wavelengths. The temporal pulses at $\lambda_{1}$ and $\lambda_{2}$ are separated by $2 / f_{\text {IM }}$. The other locations on the temporal grid are left unoccupied, probably because the two emitted wavelengths capture all the available gain. In this case, the pulsewidths are recorded to be 50 and $45 \mathrm{ps}$, yielding a time-bandwidth product of 0.56 and 0.48 , respectively. The birefringence of the IM combined with the polarizing behavior of the UMZI acts as a filter that imposes a wavelength spacing of $0.7 \mathrm{~nm}$. This could be a means of anchoring the emitted wavelengths on the ITU grid.

Conversely, one could impose a constraint in the frequency domain by inserting a periodic filter in the cavity. The use of an intracavity Fabry-Pérot filter in conjunction with an acoustooptic frequency shifter [7] might enable us to force laser oscillations at closely spaced wavelengths, still in an alternate mode.

\section{CONCLUSION}

We have demonstrated alternate pulsed operation at three wavelengths by use of a UMZI. The proposed method requires a limited number of optical components. By emitting successive pulses at different wavelengths, codes associating one time slot with one wavelength can be implemented. This might enable the implementation of dynamic 2-D OCDMA systems [12], [13]. Further work will include the stabilization of the obtained pulse trains, the control of the emitted colors/pulse temporal locations, and the increase of the number of trains and wavelengths that can be emitted at higher frequencies.

\section{REFERENCES}

[1] J. B. Schlager, S. Kawanishi, and M. Saruwatari, "Dual wavelength pulse generation using mode-locked erbium-doped fiber ring laser," Electron. Lett., vol. 27, pp. 2072-2073, 1991.

[2] J. Q. Sun and W. Liu, "Multiwavelength generation by utilizing second-order nonlinearity of $\mathrm{LiNbO}_{3}$ waveguides in fiber lasers," Opt. Commun., vol. 224, pp. 125-130, 2003.

[3] K. L. Lee and C. Shu, "Alternate and simultaneous generation of 1-GHz dual-wavelength pulses from an electrically tunable harmonically modelocked fiber laser," IEEE Photon. Technol. Lett., vol. 12, pp. 624-626, June 2000.

[4] J. Yao, Y. Wang, S. C. Tjin, Y. Zhou, Y. L. Lam, J. Liu, and C. Lu, "Active mode locking of tunable multi-wavelength fiber ring laser," Opt. Commun., vol. 191, pp. 341-345, 2001.

[5] G. E. Town, L. Chen, and P. W. W. Smith, "Dual wavelength modelocked fiber laser," IEEE Photon. Technol. Lett., vol. 12, pp. 1459-1461, Nov. 2000.

[6] G. E. Town, J. Chow, K. Sugden, I. Bennion, and M. Romagnoli, "Applications of chirped grating filters in broadband optical fiber systems," J. Electr. Electron. Eng. Aust., vol. 15, no. 3, pp. 267-276, 1995.

[7] J.-N. Maran, S. LaRochelle, and P. Besnard, "Erbium-doped fiber laser simultaneously mode locked on more than 24 wavelengths at room temperature," Opt. Lett., vol. 28, pp. 2082-2084, 2003.

[8] A. Olsson and C. L. Tang, "Time-wavelength multiplexing of modelocked external cavity semiconductor lasers," IEEE J. Quantum Electron., vol. QE-18, pp. 1982-1983, Nov. 1982.

[9] A. Yariv, Optical Electronics in Modern Communications, 5th ed. London, U.K.: Oxford Univ. Press, 1997.

[10] J. Chow, G. Town, B. Eggleton, M. Ibsen, K. Sugden, and I. Bennion, "Multiwavelength generation in an erbium-doped fiber laser using in-fiber comb filters," IEEE Photon. Technol. Lett., vol. 8, pp. 60-62, Jan. 1996.

[11] J. Sun, J. Qiu, and D. Huang, "Multiwavelength erbium-doped fiber lasers exploiting polarization hole burning," Opt. Commun., vol. 182, pp. 193-197, 2000.

[12] J. A. Salehi, "Code-division multiple-access techniques in optical fiber networks-part I: Fundamental principles," IEEE Trans. Commun., vol. 37, pp. 824-833, Aug. 1989.

[13] L. Tancevski and I. Andonovic, "Wavelength hopping/time spreading code division multiple access," Electron. Lett., vol. 30, pp. 1388-1390, 1994. 